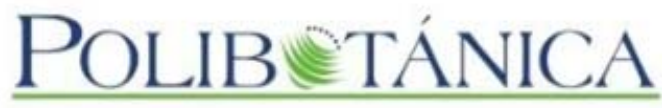

\title{
Polibotánica
}

ISSN electrónico: 2395-9525

polibotanica@gmail.com

Instituto Politécnico Nacional

México

http:www.polibotanica.mx

\section{GERMINACIÓN Y CARACTERÍSTICAS DE PLÁNTULAS DE Uspí (Couepia polyandra: Chrysobalanaceae) EN CONDICIONES DE VIVERO RÚSTICO EN CAMPECHE, MÉXICO}

\section{GERMINATIONAND \\ CHARACTERISTICS OF SEEDLINGS OF Uspí (Couepia polyandra: Chrysobalanaceae) UNDER RUSTIC NURSERY CONDITIONS IN CAMPECHE, MEXICO}

Mejenes-López, S.M.A.; G.R. Chi-Sáenz, C. Flota-Bañuelos, B. Candelaria-Martínez, y R.A. Chiquini-Medina.

GERMINACIÓN Y CARACTERÍSTICAS DE PLÁNTULAS DE Uspí (Couepia polyandra: Chrysobalanaceae) EN CONDICIONES DE VIVERO RÚSTICO EN CAMPECHE, MÉXICO. GERMINATION AND CHARACTERISTICS OF SEEDLINGS OF Uspí (Couepia polyandra: Chrysobalanaceae) UNDER RUSTIC NURSERY CONDITIONS IN CAMPECHE, MEXICO.

Polibetánica Instituto Politécnico Nacional
Núm. 48: 111-120 México. Julio 2019

DOI: $10.18387 /$ polibotanica.48.9

(c) (i) Este es un artículo de acceso abierto bajo la licencia Creative Commons 4.0 Atribución-No Comercial (CC BY-NC 4.0 Internacional). 
GERMINACIÓN Y CARACTERÍSTICAS DE PLÁNTULAS DE Uspí (Couepia polyandra: Chrysobalanaceae) EN CONDICIONES DE VIVERO RÚSTICO EN CAMPECHE, MÉXICO

\section{GERMINATION AND CHARACTERISTICS OF SEEDLINGS OF Uspí (Couepia polyandra: Chrysobalanaceae) UNDER RUSTIC NURSERY CONDITIONS IN CAMPECHE, MEXICO}

Mejenes-López, S.M.A.;

G.R. Chi-Sáenz,

C. Flota-Bañuelos,

B. Candelaria-Martínez,

R.A. Chiquini-Medina

GERMINACIÓN Y

CARACTERÍSTICAS DE

PLÁNTULAS DE Uspí

(Couepia polyandra:

Chrysobalanaceae) EN

CONDICIONES DE VIVERO

RÚSTICO EN CAMPECHE, MÉXICO

GERMINATION AND

CHARACTERISTICS OF

SEEDLINGS OF Uspí

(Couepia polyandra:

Chrysobalanaceae) UNDER

RUSTIC NURSERY

CONDITIONS IN

CAMPECHE, MEXICO

\section{POLIBETÁNICA}

Instituto Politécnico Nacional

Núm. 48: 111-120. Julio 2019

DOI:

10.18387/polibotanica.48.9 Sihochac, Champotón, Campeche, México

\author{
S. M.A. Mejenes-López / solmejenes @ hotmail.com \\ G.R. Chi-Sáenz \\ Instituto Tecnológico de Chiná, ITChiná, TecNM. Calle 11 s/n \\ entre 22 y 28 Chiná, Campeche, CP 24520. México.
}

C. Flota-Bañuelos

Catedrática CONACYT -Colegio de Postgraduados Campus Campeche. Carretera Federal Haltunchén-Edzná km 17.5,

B. Candelaria-Martínez

R.A. Chiquini-Medina

Instituto Tecnológico de Chiná, ITChiná, TecNM. Calle $11 \mathrm{~s} / n$ entre 22 y 28 Chiná, Campeche, CP 24520. México.

RESUMEN: Couepia polyandra (Kunth) Rose pertenece a la familia Chrysobalanaceae, su distribución es neotropical y es la única especie de este género de árboles que habita en México. Crece silvestre en los estados de la vertiente del Pacífico y cultivado en el Golfo hasta la Península de Yucatán. En el estado de Campeche se conoce como Uspí, produce un fruto de drupa pulposa color amarillo-naranja. El objetivo del trabajo fue determinar el efecto de tres sitios geográficos del estado de Campeche, México sobre las características morfológicas y porcentaje de germinación de semillas de Uspí, así como el desarrollo de plántulas en un vivero rústico. Se tomaron 47 semillas por cada árbol de tres sitios, S1: Chiná, Campeche, S2: La Viuda y S3: El Puente San Isidro, Palizada; a las cuales se les midió altura, ancho y peso. A las plántulas se le midió la altura, diámetro, así como la longitud y ancho de la hoja. Las semillas de las tres poblaciones presentaron diferencias significativas en el largo, ancho y peso de las semillas. Las semillas de mayor tamaño, grosor y longitud originaron plántulas más pequeñas. Las semillas S1 y S2 mostraron alta viabilidad, con un $76.6 \%$ de germinación al día 40, las semillas provenientes del S3 presentaron los valores más altos en el largo, ancho y peso; sin embargo, tuvieron menor porcentaje de germinación y las plántulas con menor superficie foliar. Por el contrario, las semillas con menor tamaño y peso provenientes de S1 y S2 tuvieron mayor porcentaje de germinación y plántulas más grandes. Dada la importancia de la planta, se deben considerar estas características, para la selección de semillas y propagación en viveros.

Palabras clave: Porcentaje de germinación, Crecimiento, Germoplasma, Correlación

ABSTRACT: Couepia polyandra (Kunth) Rose belongs to the family Chrysobalanaceae, its distribution is Neotropical and is the only species of this genus of trees that lives in Mexico. It grows wild in the states of the Pacific slope and cultivated in the Gulf to the Yucatan Peninsula. In the state of Campeche, it is known 
as Uspí, it produces a yellow-orange pulpy drupe fruit. The objective of the work was to determine the effect of three geographic sites of the state of Campeche, Mexico on the morphological characteristics and seeds germination percentage of Uspí, as well as the development of seedlings in a rustic nursery. 47 seeds were taken for each tree from three sites, S1: Chiná, Campeche, S2: La Viuda and S3: El Puente San Isidro, Palizada; to which height, width and weight were measured. The height, diameter, as well as the length and width of the leaf were measured to the seedlings. The seeds of the three populations presented significant differences in the length, width and weight of the seeds. Seeds of larger size, thickness and length originated smaller seedlings. Seeds S1 and S2 showed high viability, with $76.6 \%$ germination at day 40, the seeds from S3 presented the highest values in length, width and weight; however, they had a lower of germination percentage and seedlings with a smaller leaf surface. In contrast, seeds with smaller size and weight from S1 and S2 had a higher of germination percentage and bigger seedlings. Given the importance of the plant, these characteristics should be considered, for the selection of seeds and propagation in nurseries

Key words: Germination percentage, Growth, Germplasm, Correlation.

\section{INTRODUCCIÓN}

La planta Couepia polyandra (Kunth) Rose, presenta una distribución neotropical, abarcando la mayor parte de Mesoamérica y Costa Rica (Durán-Espinosa \& Lorea Hernández, 2010). Es originaria de México (Ojasti, 2001), y se distribuye en la vertiente del pacífico (Pennington \& Sarukhán, 2005) desde el sur de Sinaloa (Prance, 1972), Nayarit (Bravo B., Sánchez-González, de Nova V., \& Pavón H., 2016), Jalisco (González C., Aguirre C., \& Gallegos R., 2006), Colima (Padilla-Velarde, Cuevas-Guzmán, Ibarra-Manríquez, \& Moreno-Gómez, 2006), Michoacán (Cué Bär, Villaseñor, Arredondo Amezcua, Cornejo Tenorio, \& Ibarra Manríquez, 2006), Guerrero (Lozada Pérez, 2012), al noreste de Puebla (M. A. Martínez, Evangelista, Basurto, Mendoza, \& Cruz-Rivas, 2007), Oaxaca (Luna-José \& Rendón-Aguilar, 2008), Chiapas (Gutiérrez B., 2004), en la vertiente del golfo de Veracruz (Durán-Espinosa \& Lorea Hernández, 2010), Tabasco (Lascurain, Avendaño, del Amo, \& Niembro, 2010) introducido en la Península de Yucatán donde se conoce con el nombre de Uspí (Carnevali Fernández Concha, Tapia Muñoz, Duno de Stefano, \& Ramírez Morillo, 2010). Se encuentra a diferentes altitudes, que van de 0 a 600 m (Durán-Espinosa \& Lorea Hernández, 2010), así como asociadas a diferentes tipos de vegetación, tales como selvas medianas subperennifolias hasta selvas bajas caducifolias (Pennington \& Sarukhán, 2005).

Es un árbol con fuste irregular que mide de 6 a $30 \mathrm{~m}$ de alto, con un diámetro a la altura del pecho (DAP) hasta de $40 \mathrm{~cm}$, con copa dispersa, amplia y oscura. La corteza externa es lisa a ligeramente escamosa, de color verde grisáceo y la corteza interna es de color moreno rojizo intenso, sin exudado. Sus hojas de forma oblongas a oblongo-elípticas, de 6.0 a $12.5 \mathrm{~cm}$ de largo, 2.3 a $7.2 \mathrm{~cm}$ de ancho, pubescentes en el haz cuando jóvenes y glabras cuando son maduras, envés densamente aracnoides, ápice acuminado, base redondeada a cuneada, nervios prominentes en el envés de 8-15 pares. Presenta inflorescencias axilares, terminales, paniculadas, de 4-14 cm de largo, pubescentes, los pelos pardos claros. El fruto es una drupa elipsoide de 40-45 x 20-25 mm, con mesocarpo carnoso y endocarpo delgado (Lascurain et al., 2010; Pennington \& Sarukhán, 2005). Geilfus, (1994) indica que presenta una reproducción sexual, por semilla que germina de dos o tres meses en condiciones no controladas.

Dada la importancia de C. polyandra por sus diversos usos como el del fruto comestible de auto abasto y venta (Magaña Alejandro, 2010; Martínez, Evangelista, Basurto, Mendoza, \& CruzRivas, 2007; Pennington \& Sarukhán, 2005, Vázquez Torres, Campos Jiménez, Armenta Montero \& Carvajal Hernández, 2010), medicina tradicional como anticonceptivo (Biblioteca Digital de la Medicina Tradicional Mexicana, 2009), en la agroindustria, para la elaboración de velas y jabones (Vázquez Torres, Campos Jiménez, Armenta Montero, \& Carvajal Hernández, 2010), árbol para sombra y leña (Martínez, Evangelista, Basurto, Mendoza, \& Cruz-Rivas, 
2007; Biblioteca Digital de la Medicina Tradicional Mexicana, 2009). Ante esto, Flores-Guido \& Flores, (2000) la consideran como una variedad útil, tiene una fruta marginada entrando a la pérdida de germoplasma con un riesgo extremo de la extinción, lo cual implica una disminución en la capacidad de producción y de mejoramiento genético. Cabe señalar que Ruenes Morales, Montañez Escalante, Ancona, \& Ek Rodríguez, (2016) reportan a Uspí como elemento florístico de las selvas de Yucatán, cultivada de manera aislada o tolerada en los solares o huertos familiares además de ser considerado subutilizado porque su mercado es local y no se ha desarrollado un cultivo para un mercado amplio. Sin embargo, son escasos los estudios de esta especie en México. En este sentido, Vallejo-Marín, Domínguez, \& Dirzo (2006) señalan que $C$. polyandra reporta un rango amplio para la germinación de las semillas con valores de 60 a 98\%, por su parte Laines-Canepa \& Hernández-Hernández (2017) reportan una respuesta consistente de las plántulas de Uspí a fertilizantes y biofertilizantes bajo condiciones controladas y en semillas de alta calidad biológica. Martin, Velasco, Gutiérrez-García, Palacios, \& Gómez (2008) reportan una sobrevivencia de $23.4 \%$ y una altura de $36.9 \mathrm{~cm}$ en plántulas de Uspí con 12 meses de edad en condiciones de vivero. Actualmente es poca la información agronómica generada sobre $C$. polyandra, por lo que se carece de la tecnología básica para la implementación de planes estratégicos de rescate y multiplicación de la especie. El objetivo del presente trabajo fue determinar el efecto de tres sitios geográficos del estado de Campeche, México sobre las características morfológicas y porcentaje de germinación de la semilla de Uspí (C. polyandra), así como el desarrollo de plántulas en vivero rústico.

\section{MÉTODOS}

\section{Descripción del área de procedencia de las semillas}

Para la obtención de semillas, se llevó a cabo la recolecta de los frutos en agosto de 2017. Los frutos provinieron de tres individuos de diferentes sitios (tabla 1). Sitio 1 Chiná, Campeche, individuo ubicado en el Campus del Instituto Tecnológico de Chiná al sur de la ciudad de Campeche (1946'18.4" N y 90³0'08.5" O, 40 msnm), Sitio 2 La Viuda $\left(18^{\circ} 15^{\prime} 38.03 " \mathrm{~N}\right.$ y $\left.92^{\circ} 04^{\prime} 30.87^{\prime \prime} \mathrm{O}, 10 \mathrm{msnm}\right)$ y Sitio 3 El Puente San Isidro $\left(18^{\circ} 15^{\prime} 21.7^{\prime \prime} \mathrm{N}\right.$ y $92^{\circ} 05^{\prime} 16.4^{\prime \prime} \mathrm{O}, 8$ $\mathrm{msnm}$ ), localidades que pertenecen al municipio de Palizada. Los sitios se eligieron después de realizar una búsqueda del árbol para conocer sus usos y distribución en el estado de Campeche.

Tabla 1. Datos morfométricos y ambientales de los individuos nativos de la semilla de C. polyandra.

\begin{tabular}{ccccccccc}
\hline Sitios & $\begin{array}{c}\text { Alt } \\
(\mathbf{m})\end{array}$ & $\begin{array}{c}\text { Circ } \\
(\mathbf{c m})\end{array}$ & $\begin{array}{c}\text { DAP } \\
(\mathbf{c m})\end{array}$ & $\begin{array}{c}\text { Copa } \\
(\mathbf{m})\end{array}$ & Ambiente & $*$ Tm & $\begin{array}{c}* \mathbf{P P} \\
(\mathbf{m m})\end{array}$ & **TS \\
\hline 1 & 9.53 & 61.5 & 21 & 5.32 & Urbanizado & 25.7 & 1127 & Vertisol \\
2 & 10.25 & 123 & 41.5 & 8.15 & Solar con mangal & 25.3 & 1900 & Gleysol \\
3 & 5.75 & 134 & 54 & 10.22 & Solar con mangal & 25.3 & 1900 & Gleysol \\
\hline
\end{tabular}

Alt: altura, Circ: circunferencia, DAP: diámetro a la altura del pecho, Copa: Diámetro de la copa, m: metros, cm: centímetros, mm: milímetros, Tm: temperatura media anual, PP: precipitación promedio TS: tipo de suelo ( ${ }^{*}$ García, 2004, INEGI, 2010;**Bautista Zuñiga et al., 2010)

\section{Caracterización y siembra de las semillas}

El vivero rústico se estableció el 28 de agosto de 2017 en el Instituto Tecnológico de Chiná, en un área de 2x5 m, bajo sombra de un árbol llamado aguacate (Persea americana Mill.). Del total de semillas, se tomaron aleatoriamente 47 provenientes de cada individuo (S1, S2 y S3), teniendo un total de 141 unidades experimentales. Se realizó el proceso de escarificación de 
inmersión corta en agua el 25 de agosto, que consistió en mantener las semillas en un recipiente con agua y limpiar la semilla con la mano hasta remover resto de endocarpo del fruto, posteriormente a cada semilla, se le midió el ancho $(\mathrm{mm})$ y la longitud $(\mathrm{mm})$ con un vernier digital marca Traceable, después se dejaron secar a sombra sobre una charola de aluminio de base plana, a temperatura ambiente durante $24 \mathrm{~h}$, para posteriormente determinar su peso con una báscula digital de alta precisión SF400 10kg/1g.

Antes de la siembra, el germoplasma se remojó en un recipiente de plástico con agua potable durante 48 h. subsiguiente ( 26 y 27 agosto), después de transcurrido ese tiempo, se realizó la siembra directa ( 28 de agosto) en bolsas de uso forestal de polietileno color negro $(20 \times 20 \mathrm{~cm})$, colocando la semilla a $5 \mathrm{~cm}$ de profundidad en tierra negra de origen vertisol, que no recibió tratamiento, procedente de la selva mediana subcaducifolia con vegetación secundaria (Bautista, Zuñiga et al., 2010) con textura del suelo de clase franco-arcillosa arenosa, medianamente alcalino ( $\mathrm{pH} 8.12$ ), con un porcentaje de materia orgánica muy bajo (1.7), con una conductividad eléctrica salina (4.79 dS). Se regaban con agua corriente cada tercer día durante la tarde después de las 16:00 h.

\section{Porcentaje de germinación}

Las semillas sembradas fueron revisadas diariamente durante 40 días (del 28 de agosto al 6 de octubre de 2017), definiéndose como el periodo cuando se alcanza la máxima tasa de germinación, para posteriormente evaluar la germinación. Para evaluar el porcentaje de germinación, se empleó la fórmula $\% G=\frac{\mathrm{np}(100)}{47}$, donde: $\% \mathrm{G}=$ porcentaje de germinación, $\mathrm{np}=$ número de plántulas germinadas y 47 corresponde al número total de semillas usadas para la siembra.

\section{Longitud del tallo, diámetro basal, número de hojas y ancho y longitud de la hoja}

Cada cinco días a cada plántula se le midió la altura del tallo desde la base (superficie del sustrato) hasta el ápice, que fue considerado donde crece la base de la hoja más joven. Se tomó el criterio para la obtención del diámetro del tallo a $5 \mathrm{~cm}$ de su altura a partir de la base. Durante la toma de datos se empleó un vernier marca Traceable y una regla graduada $(10 \mathrm{~mm}=$ $1 \mathrm{~cm})$. Se contó el número de hojas por plántula y finalmente se realizó la medida del ancho y longitud de la hoja más grande, con la misma regla graduada. La toma de datos se realizó después de los 15 días de la siembra, a partir del 12 de septiembre hasta el 30 de octubre, haciendo un total de 48 días.

\section{Análisis estadístico}

Para conocer el efecto del árbol de origen con las características de las semillas, se realizó un análisis paramétrico, bajo un diseño completamente al azar, con una comparación de medias con Tukey ( $\mathrm{P} \leq 0.05)$. Para determinar la viabilidad de las semillas con relación a la plántula emergida, se realizó un análisis de varianza con mediciones repetidas en el tiempo y con una comparación de medias con Tukey $(\mathrm{P} \leq 0.05)$. Los datos de altura de la semilla, diámetro de la semilla, peso de la semilla y altura de plántula se correlacionaron bajo un análisis no paramétrico, Spearman $(\mathrm{P} \leq 0.05)$. Los datos se analizaron con el paquete estadístico STATISTICA V.7.1, (Start Soft, 2005).

\section{Resultados}

\section{Morfología de Semillas}

Las semillas de las tres poblaciones presentaron diferencias significativas en el largo $\left(\mathrm{F}_{140,1}=38.13\right.$, $\mathrm{P} \leq 0.05$ ), siendo más largas las semillas del $\mathrm{S} 3$ con una media de $39.90 \mathrm{~mm}$, seguidas de $\mathrm{S} 1$ y S2, con 38.13 y $33.77 \mathrm{~mm}$, respectivamente. Las semillas más anchas $\left(\mathrm{F}_{140,1}=17.53, \mathrm{P} \leq 0.05\right)$ se encontraron en el S3 y S1, con 22.47 y $21.66 \mathrm{~mm}$. Con relación al peso de las semillas, las más pesadas $\left(\mathrm{F}_{140,1}=24.469, \mathrm{P} \leq 0.05\right)$ se encontraron en el $\mathrm{S} 3$ y S1 con 14.65 y 13.27 g. (tabla 2$)$. 
Tabla 2. Características morfológicas de las semillas provenientes de S1 (Chiná), S2 (La Viuda) y S3 (El Puente San Isidro) Campeche, México.

\begin{tabular}{cccccccc}
\hline Sitio & \multirow{2}{*}{ n } & \multicolumn{2}{c}{ Largo } & \multicolumn{2}{c}{ Ancho } & \multicolumn{2}{c}{ Peso } \\
& & mm & $\begin{array}{c}\text { Std. } \\
\text { Dev }\end{array}$ & mm & $\begin{array}{c}\text { Std. } \\
\text { Dev. }\end{array}$ & g & $\begin{array}{c}\text { Std. } \\
\text { Dev. }\end{array}$ \\
\hline 1 & 47 & $38.13^{\mathrm{a}}$ & 4.13 & $21.66^{\mathrm{ab}}$ & 2.79 & $13.27^{\mathrm{a}}$ & 4.49 \\
2 & 47 & $33.77^{\mathrm{b}}$ & 3.01 & $19.74^{\mathrm{b}}$ & 1.71 & $9.97^{\mathrm{a}}$ & 2.44 \\
3 & 47 & $39.90^{\mathrm{a}}$ & 3.25 & $22.465^{\mathrm{a}}$ & 2.23 & $14.65^{\mathrm{a}}$ & 2.67 \\
Total & 141 & 37.26 & 4.33 & 21.29 & 2.54 & 12.63 & 3.85 \\
\hline
\end{tabular}

${ }^{\mathrm{a}, \mathrm{b}}$ Literales distintas en las misma columna indican diferencias significativas, $\mathrm{P} \leq 0.05$

Relación semilla, plántula y porcentaje de germinación

Se observó que, de las semillas con características morfológicas mayores, como son peso $(\mathrm{rs}=-0.299044)$, grosor $(\mathrm{rs}=-0.240429)$ y longitud $(\mathrm{rs}=-0.285626)$, emergieron plántulas más pequeñas. De manera general, las semillas más pequeñas presentaron mayor viabilidad, al presentar mayor porcentaje de germinación, con $76.6 \%$ de semillas germinadas al día 40, siendo éstas S1 y S2 (fig. 1).

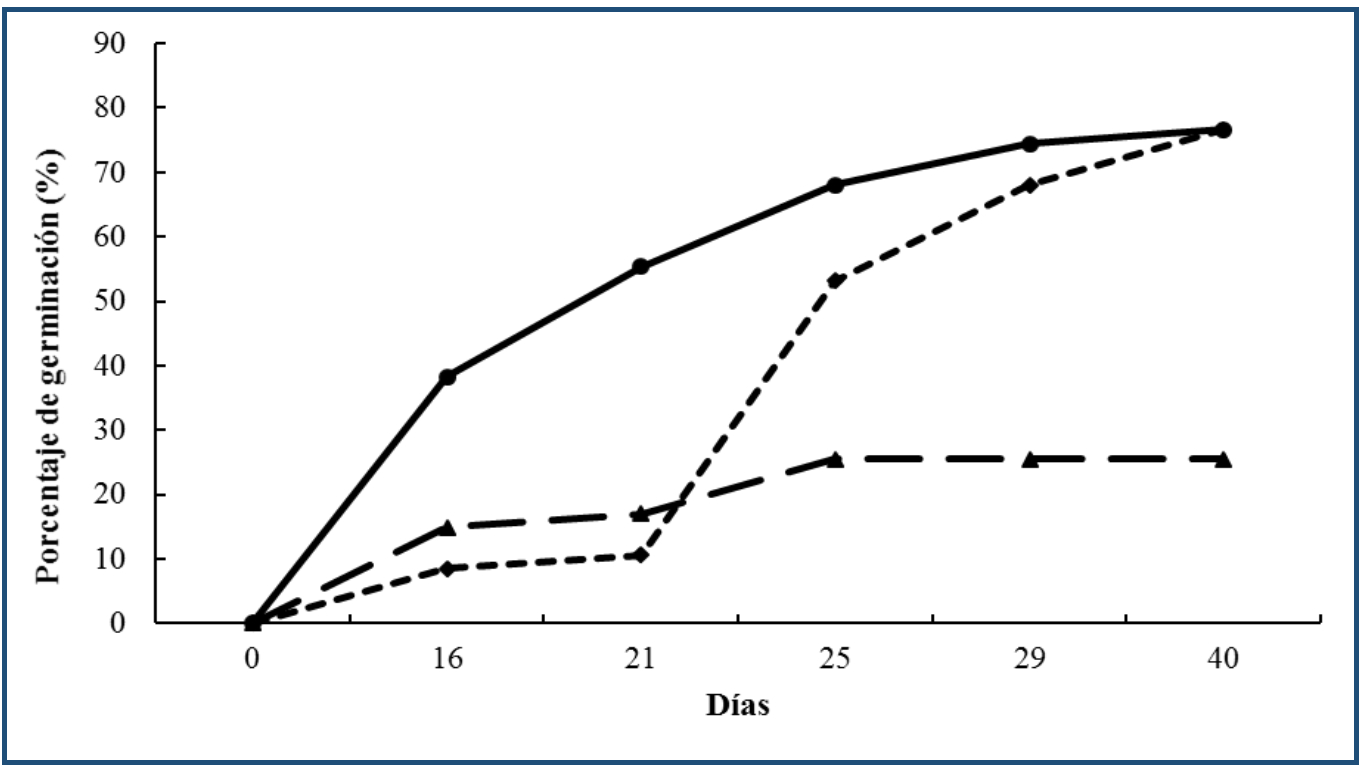

Fig. 1. Porcentaje de germinación de las semillas de $C$. polyandra bajo condiciones de vivero rústico.

$$
\mathrm{S} 1:-\bullet-\mathrm{S} 2 \text { : }
$$$$
\longrightarrow \text { y S3: }
$$ 


\section{Longitud del tallo, diámetro basal y número de hojas}

Las plántulas con mayor altura $\left(\mathrm{F}_{140,1}=22.9611\right.$, P P $\left.\leq 0.05\right)$, fueron las provenientes de S1 y $\mathrm{S} 2$ con alturas promedio de 121.93 y $165.46 \mathrm{~mm}$. El mayor diámetro del tallo se registró en las plántulas de $\mathrm{S} 2\left(\mathrm{~F}_{140,1}=23.8353, \mathrm{P} \leq 0.05\right)$ con $1.954 \mathrm{~mm}$. Las plántulas con las hojas más largas $\left(F_{140,1}=52.3063, P \leq 0.05\right)$, crecieron en el $S 2$, seguido del S1 y el S3 con 81.06, 56.34 y $8.676 \mathrm{~mm}$, respectivamente. Las plántulas con las hojas más anchas $\left(\mathrm{F}_{140,1}=30.2072, \mathrm{P} \leq 0.05\right)$ fueron las del S2 y S1, seguidas del S3 con 29.92, 26.46 y $3.94 \mathrm{~mm}$. Finalmente se observó que las plántulas de S1 y $\mathrm{S} 2$, tuvieron mayor foliar $\left(\mathrm{F}_{140,1}=21.2804, \mathrm{P} \leq 0.05\right)$ con 815.17 y 615.25 $\mathrm{m}^{2}$ en promedio respectivamente (tabla 3 ).

Tabla 3. Altura de la plántula, diámetro del tallo, el largo, ancho, número de hojas de las plántulas y área foliar por cada sitio.

\begin{tabular}{|c|c|c|c|c|c|c|c|c|c|c|c|c|c|}
\hline \multirow{2}{*}{$\mathbf{S}$} & \multirow{2}{*}{$\mathbf{N}$} & \multicolumn{2}{|c|}{ Altura } & \multicolumn{2}{|c|}{$\begin{array}{l}\text { Diámetro } \\
\text { del tallo }\end{array}$} & \multicolumn{2}{|c|}{ Largo } & \multicolumn{2}{|c|}{ Ancho } & \multicolumn{2}{|c|}{$\begin{array}{l}\text { Número } \\
\text { de hojas }\end{array}$} & \multicolumn{2}{|c|}{ Superficie foliar } \\
\hline & & $\mathbf{m m}$ & $\begin{array}{l}\text { Std. } \\
\text { Dev }\end{array}$ & $\mathbf{m m}$ & $\begin{array}{l}\text { Std. } \\
\text { Dev }\end{array}$ & $\mathbf{m m}$ & $\begin{array}{l}\text { Std. } \\
\text { Dev. }\end{array}$ & $\mathbf{m m}$ & $\begin{array}{l}\text { Std. } \\
\text { Dev. }\end{array}$ & $\bar{x}$ & $\begin{array}{l}\text { Std. } \\
\text { Dev. }\end{array}$ & $\mathbf{m}^{2}$ & Std. Dev. \\
\hline 1 & 47 & $121.93^{\mathrm{b}}$ & 84.57 & $1.34^{\mathrm{b}}$ & 0.96 & $56.34^{\mathrm{b}}$ & 42.07 & $26.46^{\mathrm{a}}$ & 20.56 & $2.51^{\mathrm{a}}$ & 1.85 & $615.25^{\mathrm{a}}$ & 657.31 \\
\hline 2 & 47 & $165.46^{\mathrm{a}}$ & 68.52 & $1.95^{\mathrm{a}}$ & 0.87 & $81.06^{\mathrm{a}}$ & 36.34 & $29.92^{\mathrm{a}}$ & 19.78 & $3.31^{\mathrm{a}}$ & 1.51 & $815.17^{\mathrm{a}}$ & 634.87 \\
\hline 3 & 47 & $53.93^{\mathrm{c}}$ & 86.91 & $0.61^{\mathrm{c}}$ & 0.97 & $8.67^{\mathrm{c}}$ & 23.61 & $3.94^{\mathrm{b}}$ & 10.68 & $1.17 \mathrm{~b}$ & 1.94 & $82.38^{\mathrm{b}}$ & 339.9 \\
\hline Total & 141 & 113.78 & 92.17 & 1.30 & 1.08 & 48.69 & 45.91 & 20.11 & 20.94 & 2.33 & 1.98 & 504.27 & 639.30 \\
\hline
\end{tabular}

a,b,c Literales distintas en las misma columna indican diferencias significativas, $\mathrm{P} \leq 0.05$.

\section{DISCUSIÓN}

Las semillas provenientes del sitio 3 El Puente San Isidro, Palizada, Campeche, presentaron los valores más altos en el largo, ancho y peso de las semillas, sin embargo, tuvieron menor porcentaje de germinación y las plántulas más pequeñas con menor superficie foliar. Por el contrario, las semillas con menor tamaño y peso provenientes de Chiná, Campeche y La Viuda, Palizada (sitio 1 y 2 respectivamente), tuvieron mayor porcentaje de germinación y plántulas más grandes similar a lo obtenido Vázquez, García, Peña, Ramírez, \& Morales (2011) quienes reportan que las semillas más pequeñas en el caso de Agave salviana tuvieron mayor emergencia inicial. La altura de las plántulas fue hasta $165.46 \mathrm{~mm}$, con diámetro promedio de $1.954 \mathrm{~mm}$ a los 48 días. Siendo menores a los reportados por Laines-Canepa \& HernándezHernández (2017) obteniendo que el crecimiento va desde $232.7 \mathrm{~mm}$ hasta 329.8 en un lapso de cinco meses. Con relación al diámetro del tallo, los valores obtenidos son similares a los reportados en plántulas evaluadas por Laines-Canepa \& Hernández-Hernández (2017) con medidas que van de 1 a $3 \mathrm{~mm}$. En este estudio, se presentó el 76.6\% de germinación al día 40, coincidiendo con lo reportado por Vallejo-Marín, M., Domínguez, C.A. \& Dirzo, R.(2006) en semillas de $C$. polyandra originarias de Veracruz, quienes mencionan que la probabilidad máxima de germinación en esta especie va de 0.6 a $0.98 \%$. También indican que es una planta con una capacidad aparente para resistir la depredación (hasta 10\% de eliminación de la masa de semillas). Sin embargo, resulta inferior al compararse con Cordia subcordata, quién registra 98\% de germinación después de 34 días (Naranjo Morán \& Chica Martínez, 2017). 


\section{CONCLUSIONES}

El largo, ancho y peso de las semillas de $C$. polyandra se vieron afectadas por el sitio de origen, fueron mayores las obtenidas de un árbol de menor altura, mayor diámetro de tallo a la altura del pecho (DAP) y mayor diámetro de copa que se localizaba en un suelo tipo Gleysol con precipitación anual de 1,900 $\mathrm{mm}$. Las semillas de menor tamaño y peso presentaron un mayor porcentaje de germinación y sus plántulas presentaron un mayor crecimiento en condiciones de vivero rústico que las plántulas obtenidas de semillas de mayor tamaño y peso. Por lo tanto, para el desarrollo de programas de producción de plántulas de $C$. polyandra, deben preferirse semillas de menor tamaño.

\section{AGRADECIMIENTOS}

Al Tecnológico Nacional de México (TecNM) por el financiamiento del Proyecto n004r8 (1604). A los estudiantes Rubén Cab-Quijano, Abraham Hernández-Martínez, Viviana Miss M., Frida Santos A., Clarissa Chávez Ch., Andrea Vázquez P., Jorge Uco P. y Héctor López C., por el apoyo en el trabajo de campo y de vivero. A la señora Ricarda Pastora Mejenes Quijano por facilitarnos el hospedaje y equipo; a los pobladores del municipio de Palizada por sus conocimientos y aportaciones para el desarrollo del trabajo de campo y a la familia Magaña Morales por sus diversos apoyos durante el desarrollo del trabajo de campo en el municipio de Palizada. Finalmente, a los revisores por sus atinados comentarios y sugerencias.

\section{LITERATURA CITADA}

Bautista Zuñiga, F., Palacio Aponte, A. G., Mendoza Vega, J., Kú Quej, V. M., Pool Novelo, L., \& Cantarell Jiménez, W. (2010). Suelo. In y J. M. V. (Coord. . Villalobos-Zapata, G. J. (Ed.), La Biodiversidad en Campeche: Estudio de Estado. (1a., pp. 20--28). Campeche: Comisión Nacional para el Conocimiento y Uso de la Biodiversidad (CONABIO), Gobierno del Estado de Campeche, Universidad Autónoma de Campeche, El Colegio de la Frontera Sur.

Biblioteca Digital de la Medicina Tradicional Mexicana. (2009). Biblioteca Digital de la Medicina Tradicional Mexicana. Retrieved from http://www.ru.tic.unam.mx/handle/123456789/1549

Bravo B., O., Sánchez-González, A., de Nova V., J. A., \& Pavón H., N. P. (2016). Composición y estructura arbórea y arbustiva de la vegetación de la zona costera de Bahía de Banderas, Nayarit, México. Botanical Sciences, 94(3), 603--623.

Carnevali Fernández Concha, G., Tapia Muñoz, J. L., Duno de Stefano, R., \& Ramírez Morillo, I. M. (2010). Flora ilustrada de la Península de Yucatán: listado florístico (Centro de Investigación Científica de Yucatán, ed.). Mérida, Yucatán, México.

Cué Bär, E. M., Villaseñor, J. L., Arredondo Amezcua, L., Cornejo Tenorio, G., \& Ibarra Manríquez, G. (2006). La flora arbórea de Michoacán, México. Boletín de La Sociedad Botánica de México, 78, 47-81. Retrieved from http://www.redalyc.org/articulo.oa?id=57707806

Durán-Espinosa, C., \& Lorea Hernández, F. G. (2010). Chrysobalanaceae. Flora de Veracruz, $150,39$.

Flores-Guido, J. S., \& Flores, A. G. (2000). Los frutales del área Maya Yucateca y su importancia en la dieta alimenticia. Revista de la Universidad Autónoma de Yucatán (Vol. 214). Revista de la Universidad Autónoma de Yucatán.

García, E. (2004). Modificaciones al sistema de clasificación climática de Köppen: para adaptarlo a las condiciones de la República Mexicana (Instituto). Retrieved from https://www.academia.edu/12911044/Modificaciones_al_sistema_de_clasificación_cli mática_de_Köppen_para_adaptarlo_a_las_condiciones_de_la_República_Mexicana_2 004_._Enriqueta_García 
Geilfus, F. (edit. ). (1994). El árbol al servicio del agricultor. Manual de agroforestería para el desarrollo rural. In Guía de especies. CATIE, ENDA-CARIBE. Retrieved from http://www.manioc.org/gsdl/collect/recherch/import/tramil/arbolt1.pdf

González C., G. A., Aguirre C., O. A., \& Gallegos R., A. (2006). Caracterización Estructural del Estrato Arbóreo en un Bosque Tropical Subcaducifolio de la Costa de Jalisco. In Avances en la Investigación Científica en el CUCBA XVII Semana de la Investigación Científica (pp. 82-91). Guadalaja, Jalisco.: Orgánica Diseño Editorial.

Gutiérrez B., C. (2004). Listado florístico del Norte de Chiapas: Catazajá y límites con Palenque. Polibotánica, 17, 107-124.

INEGI. (2010). Instituto Nacional de Estadística y Geografía. Retrieved from http.www.inegi.gob.mx

Laines-Canepa, J. R., \& Hernández-Hernández, L. (2017). Evaluación del efecto de biofertilizantes y fertilizante químico sobre el crecimiento de USPÍ (Couepia polyandra) y Caracolillo (Ormosia macrocalix). Journal of Energy, Engineering, Optimization and Sustainability, 1(1), 35-50. https://doi.org/10.19136/jeeos.a1n1.1733

Lascurain, M., Avendaño, S., del Amo, S., \& Niembro, N. (2010). Guía de frutos silvestres comestibles en Veracruz. Fondo Sectorial para la Investigación, el Desarrollo y la Innovación Tecnológica Forestal, Conafor-Conacyt.

Lozada Pérez, L. (2012). Flora de Guerrero. Chrysobalanaceae. Flora de Guerrero, 5-25.

Luna-José, A. de L., \& Rendón-Aguilar, B. (2008). Recursos vegetales útiles en diez comunidades de la Sierra Madre del Sur, Oaxaca, México. Polibotánica, (26), 193242.

Magaña Alejandro, M. A. (2010). Vegetación y flora del municipio de Paraíso. In Vegetación y flora del municipio de Paraíso (1 ${ }^{\text {a }}$ edición). Villahermosa, Tabasco: Comité Editorial Divisional.

Martin, R., Velasco, E., Guetiérrez-García, G., Palacios, R., \& Gómez, B. (2008). El Parque Ecológico Jaguaroundi. In Y. Nava \& I. Rosas (Eds.), El Parque Ecológico Jaguaroundi Conservación de la selva tropical veracruzana en una zona industrializada. México: Programa Universitario de Medio Ambiente (UNAM), Secretaría de Medio Ambiente y Recursos Naturales (SEMARNAT), Instituto Nacional de Ecología (SEMARNAT).

Martínez, M. A., Evangelista, V., Basurto, F., Mendoza, M., \& Cruz-Rivas, A. (2007). Flora útil de los cafetales en la Sierra Norte de Puebla, México. Revista Mexicana de Biodiversidad, 78(1), 15-40.

Martínez, Mi. A., Evangelista, V., Basurto, F., Mendoza, M., \& Cruz-Rivas, A. (2007). Flora útil de los cafetales en la Sierra Norte de Puebla, México Useful plants of the Sierra Norte de Puebla , Mexico. Revista Mexicana de Biodiversidad, 78, 15-40.

Naranjo Morán, J., \& Chica Martínez, E. (2017). Germinación y crecimiento inicial de plántulas de Guaijí (Couepia subcordata) bajo condiciones de vivero. Bionatura, 2(2), 296-298. https://doi.org/10.21931/rb/2017.02.02.4

Ojasti, J. (2001). Especies exóticas invasoras. Estrategia regional de biodiversidad para los países del trópico andino. (p. 63). p. 63.

Padilla-Velarde, E., Cuevas-Guzmán, R., Ibarra-Manríquez, G., \& Moreno-Gómez, S. (2006). Riqueza y biogeografía de la flora arbórea del estado de Colima, México. Revista Mexicana de Biodiversidad, 77 (2), 271-295.

Pennington, T. D., \& Sarukhán, J. (2005). Árboles tropicales de México. Manual para la identificación de las principales especies ( $3^{\mathrm{a}}$ ed.). México D. F.: Fondo de Cultura Económica. México.

Prance, G. T. (1972). Chrysobalanaceae. Flora Neotropica Monograph 9, 9, 409.

Ruenes Morales, M. del R., Montañez Escalante, P. I., Ancona, J. J., \& Ek Rodríguez, I. L. (2016). Los frutales abandonados y subutilizados en la Península de Yucatán. Universidad Autónoma de Yucatán, Red temática sobre el Patrimon. Red temática sobre el Patrimonio Biocultural de México.

StatSoft; Inc. (2005). STATISTICA. Retrieved from www.statsoft.com

Vallejo-Marín, M., Domínguez, C., \& Dirzo, R. (2006). Simulated seed predation reveals a 


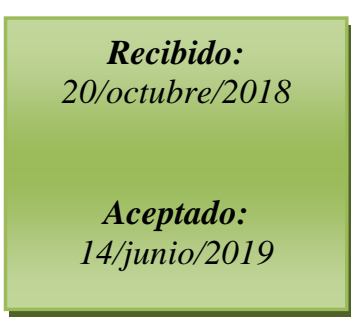

variety of germination responses of neotropical rain forest species. American Journal of Botany, 3((3)), 369-376. https://doi.org/10.3732/ajb.93.3.369

Vázquez, D. E., García, N. R., Peña, V. C. B., Ramírez, T. H. M., \& Morales, R. V. (2011). Seed size, emergence and seedling development of maguey (Agave salviana Otto ex Salm-Dyck). Revista Fitotecnia Mexicana, 34((3)), 167-173.

Vázquez Torres, M., Campos Jiménez, J., Armenta Montero, S., \& Carvajal Hernández, C. I. (2010). Árboles de la región de Los Tuxtlas. Gobierno del Estado de Veracruz, Secretaría de Educación del Estado de Veracruz. Xalapa, Veracruz. 424 págs. Retrieved

from https://www.sev.gob.mx/servicios/publicaciones/colec_veracruzsigloXXI/ArbolesTuxt la.pdf 